\title{
Beneficial triple-site cardiac resynchronization in a patient supported with an intra-aortic balloon pump for end-stage heart failure
}

\author{
Jan Ciszewski, Aleksander Maciąg, Katarzyna Gepner, Edyta Smolis-Bąk, Maciej Sterliński \\ Institute of Cardiology, Warsaw, Poland
}

Postep Kardiol Inter 2014; 10, 1 (35): 40-42 DOI: $10.5114 /$ pwki.2014.41467

\begin{abstract}
A bstract
The authors present the case of a 62-year-old male patient with an implantable cardioverter-defibrillator and end-stage heart failure supported with an intra-aortic balloon pump. Implantation of a triple-site cardiac resynchronization system and complex heart failure treatment brought a significant improvement, return to home activity and 17-month survival. The patient died due to heart failure aggravation. Within this time he was rehospitalized and successfully treated twice for an electrical storm.
\end{abstract}

Key words: end-stage heart failure, triple-site resynchronization.

\section{Introduction}

Cardiac resynchronization therapy (CRT) is a recognized method in chronic heart failure (CHF) patients. In NYHA class IV, resynchronization is recommended now only in ambulatory patients. However, there is considered to be a gap in the evidence and in some groups with serious heart failure CRT brings a beneficial effect [1, 2]. An extended cardiac resynchronization therapy has been described to pace ventricles from three different sites: from one right ventricle lead and two left ventricular (LV) leads. The feasibility and clinical benefits of triple site ventricular pacing (TriV) vs. standard CRT were described [3]. The pathophysiological background for TriV pacing suggests extensive spatial dyssynchrony reversal, which may be applicable to end-stage CHF patients in whom classical device-based therapy fails.

We present a case of TriV system use in an end-stage CHF subject supported with an intra-aortic balloon pump (IABP).

\section{Case report}

A 62-year-old patient with CHF after anterior and posterior wall myocardial infarction (MI) with a dual chamber cardioverter-defibrillator (ICD), after two percutaneous coronary interventions, was admitted to the department due to significant CHF progression and recurrent ventricular tachycardias (VTs) resulting in numerous ICD discharges. On admission, the patient presented with pulmonary congestion, peripheral edema and systolic arterial blood pressure permanently $<80 \mathrm{~mm} \mathrm{Hg}$. Left ventricle ejection fraction (LVEF) was $10 \%$ approximately and post MI left bundle branch-like block with QRS time 180 ms was present. Due to the potential proarrhythmic effect inotropic agents were found to be dangerous in arrhythmic storm and an IABP was inserted. An IABP support, loop diuretics and amiodarone infusion provided transient circulation stability without VT recurrence. Then, a decision was made to upgrade the patient's ICD to a TriV system. Two bipolar LV leads were implanted in the posterolateral and great cardiac veins; pacing thresholds were $2.8 \mathrm{~V}$ and $5.0 \mathrm{~V}$ with respect for leads' stability and the leads were switched to a $Y$ bipolar connector (the position of system leads is shown in Figure 1, native and paced electrograms are shown in Figures 2 and 3 respectively). A defibrillation test was not done as contraindicated in NYHA IV CHF. No complications were observed and the patient's functional status improved. The IABP was removed four days after the procedure. Oral pharmacotherapy and gradual smooth exercise training were introduced. The patient left the intensive care unit on day 11. Three weeks later he was discharged home with NYHA class II/III and he joined a cardiac ambulatory rehabilitation 

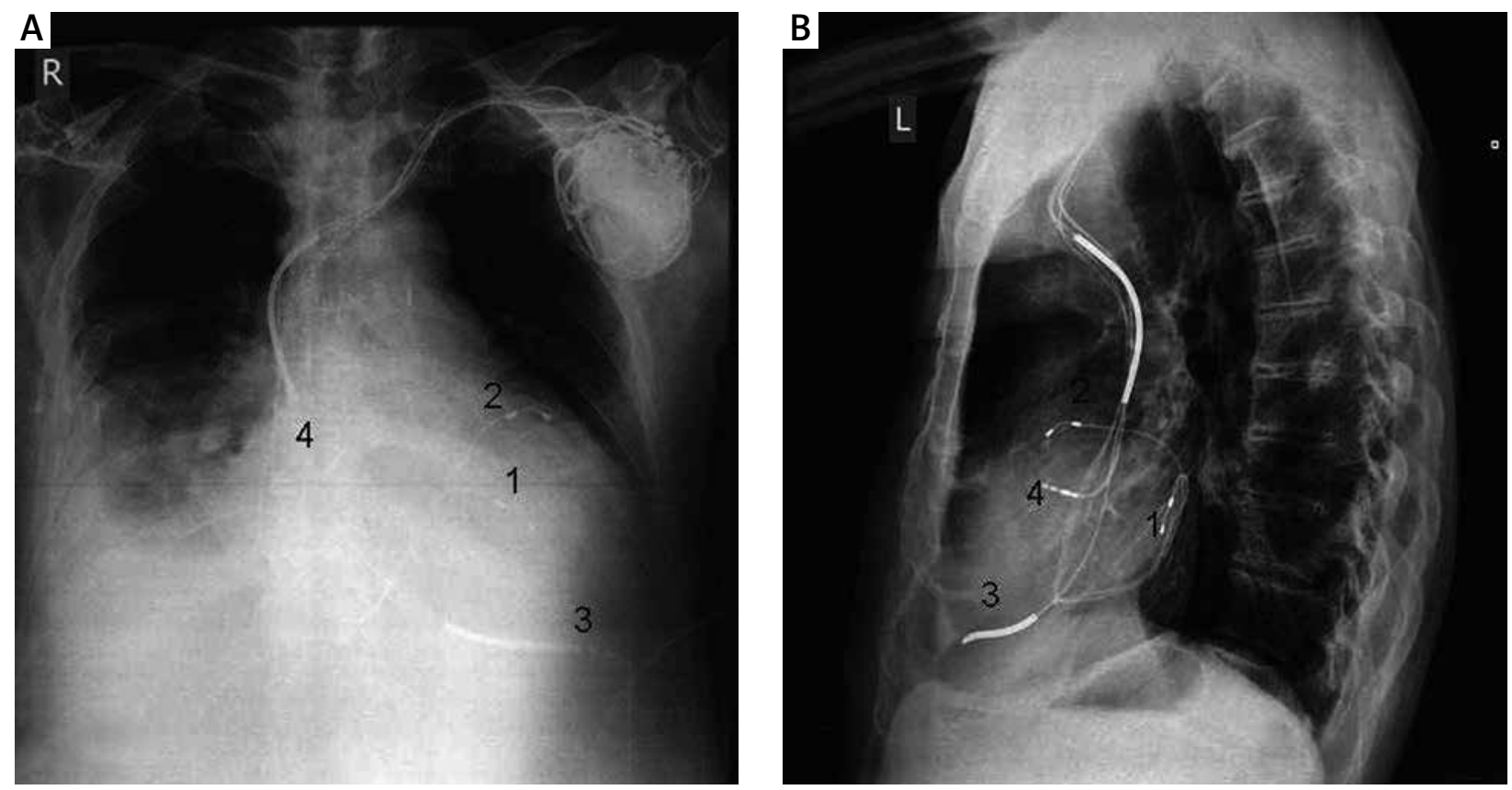

Figure 1. Final position of leads for triple site cardiac resynchronization. X-ray chest: A - postero-anterior view; B - left lateral view. Leads in 1. posterolateral vein, 2. great cardiac vein, 3. right ventricular apex and 4. right atrium appendage

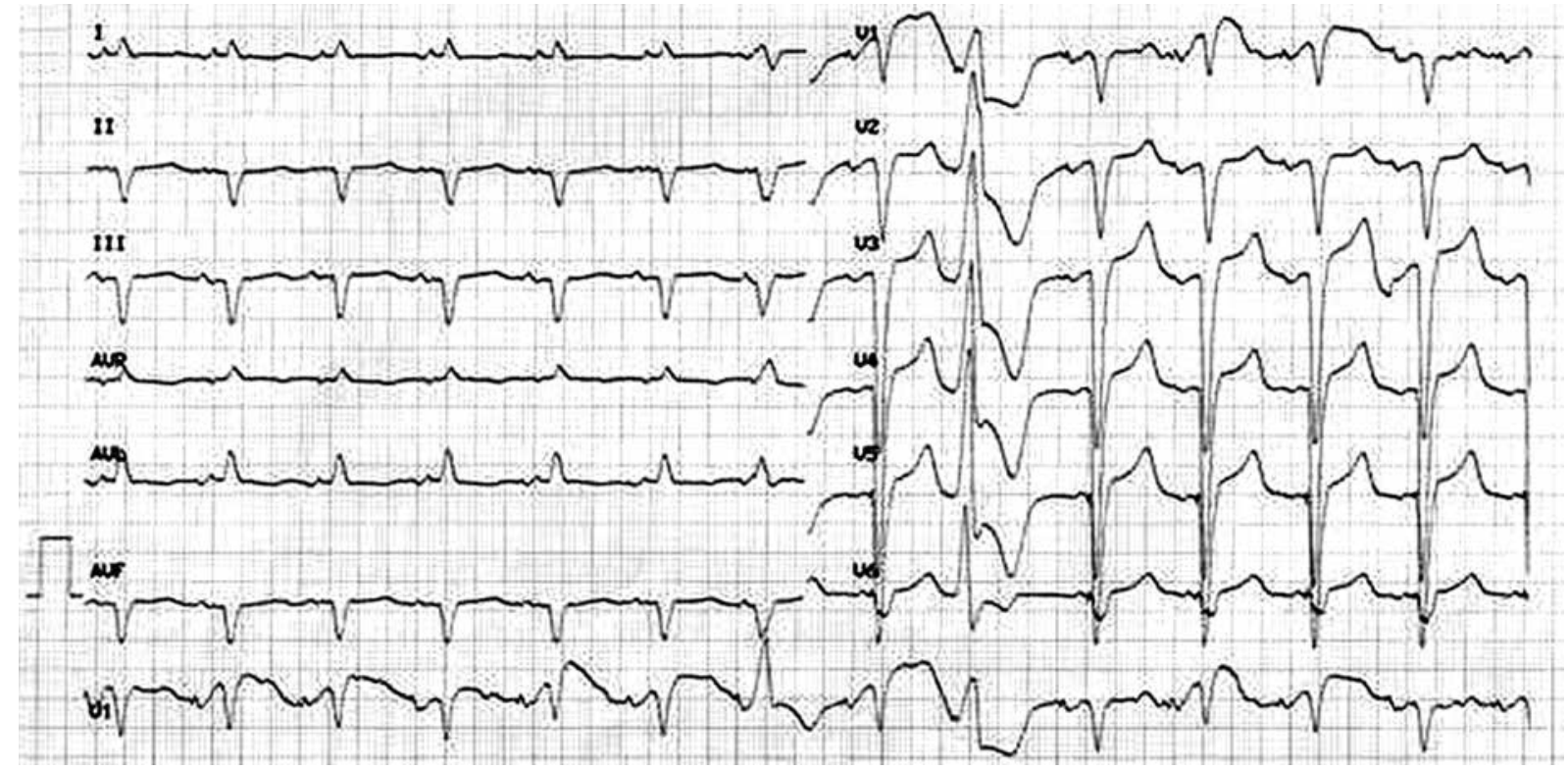

Figure 2. Patient's native ECG on admission

program subsequently. Peak $\mathrm{VO}_{2}=10.4 \mathrm{ml} / \mathrm{kg} / \mathrm{min}$ increased to $12.3 \mathrm{ml} / \mathrm{kg} / \mathrm{min} 8$ months later and LVEF increased within the same period from $10 \%$ to $30 \%$. Nine and ten months after the TriV implantation the patient developed two electrical storm (ES) events and was urgently re-hospitalized, treated successfully with intravenous amiodarone, isotonic fluid and electrolyte administration and empiric use of mexiletine p.o. Because of battery depletion due to numerous discharges, the device was electively replaced 1 year after TriV implantation. Pacing parameters remained stable. The patient died due to heart failure progression 17 months after the TriV method had been introduced.

The patient was treated according to inclusion criteria and written informed consent in the project supported by a grant of the Institute of Cardiology, Warsaw, no. 2.28/ IV/09, Ethics Committee approval IK NP-002180/1106/08. 


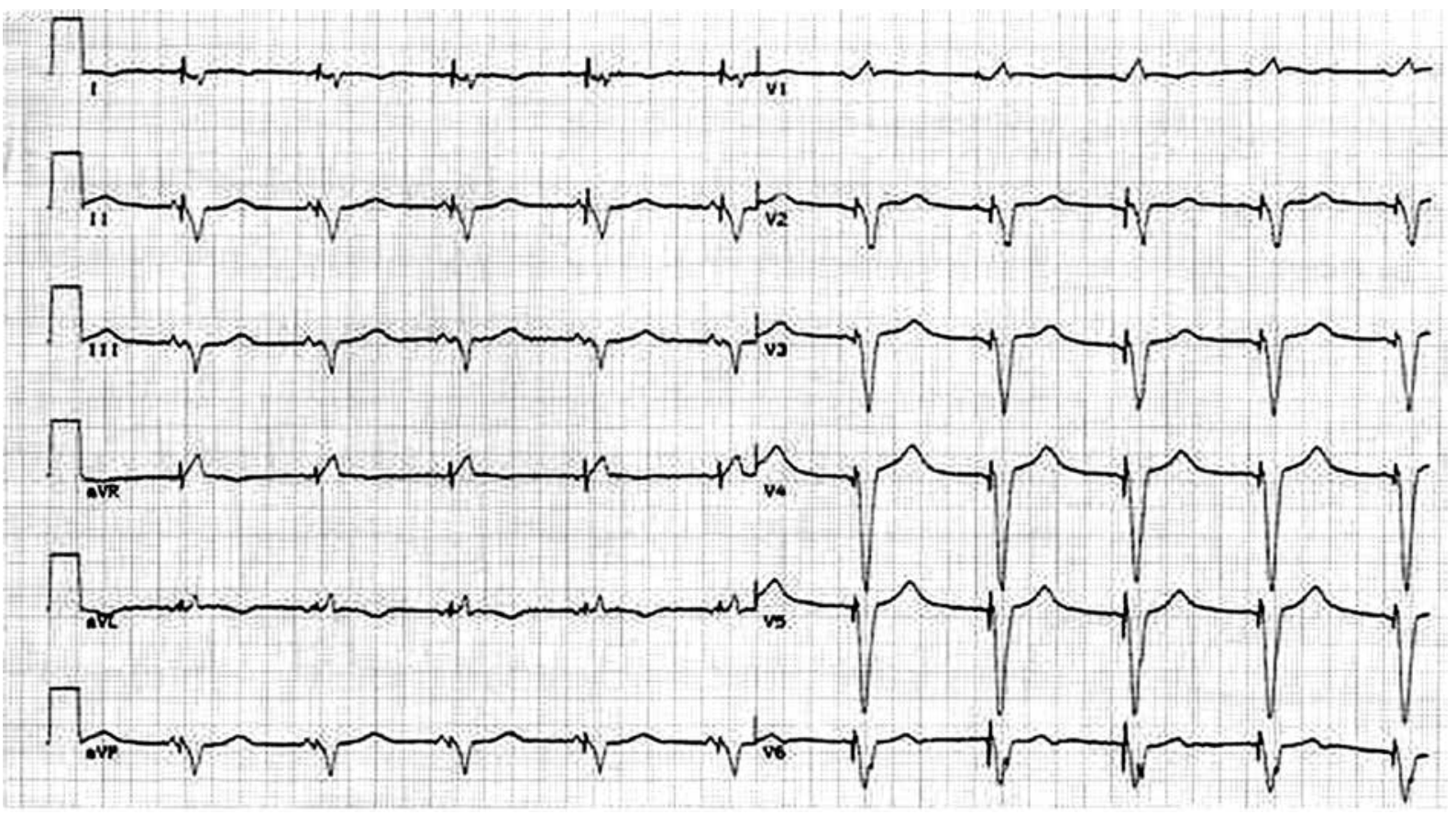

Figure 3. Paced ventricular electrogram after triple-site resynchronization system has been introduced

\section{Discussion}

The TriV pacing can be considered as a potent method for CHF patients who meet resynchronisation criteria. The currently ongoing large TRUST CRT trial, comparing TriV to conventional CRT, showed both methods to be equally safe and feasible [3, 4]. The TriV implantation may be the choice for those CHF patients who are not good candidates for conventional CRT - not supported by evidence or not responding to the therapy $[5,6]$. In the described case TriV implantation in a "no-option" IABP patient, despite slightly marked ECG changes (as shown in Figures 2 and 3), resulted in discharge home and return to limited everyday life activity. The 17-month survival is an unexpectedly long period and two re-hospitalizations within this time seem to be legitimated.

Defibrillation function is very disputable in this subject, but ICD down-grading would be controversial as well. Moreover, there was a high risk of recurrence of ventricular arrhythmias, which were eventually observed within the follow-up. The life-threatening ES events were successfully managed and the supportive role of TriV pacing was sustained. The potential relation between ES, heart failure and TriV pacing remains unclear. High energy consumption for numerous evoked shocks and dual-site LV pacing resulted in 12-month battery longevity only, which can be justified in this rare case.

Besides the device therapy, a complex approach, including lifestyle modification, optimal pharmacotherapy and gradual physical training, should be found to be important.

\section{Conclusions}

Triple-site ventricular pacing may be a therapeutic option for selected end-stage heart failure patients. Use and compliance for all the concomitant strategies of heart failure management should be emphasized.

\section{References}

1. Authors/Task Force Members. Brignole M, Auricchio A, Baron-Esquivias G, et al. 2013 ESC Guidelines on cardiac pacing and cardiac resynchronization therapy. The Task Force on cardiac pacing and resynchronization therapy of the European Society of Cardiology (ESC). Developed in collaboration with the European Heart Rhythm Association (EHRA). Eur Heart J 2013; 34: 2281-2329.

2. Castel MA, Magnani S, Mont L, et al. Survival in New York Heart Association class IV heart failure patients treated with cardiac resynchronization therapy compared with patients on optimal pharmacological treatment. Europace 2010; 12: 1136-1140.

3. Lenarczyk R, Kowalski O, Kukulski T, et al. Mid-term outcomes of triple-site vs. conventional cardiac resynchronization therapy: a preliminary study. Int J Cardiol 2009; 133: 87-94.

4. Leclercq C, Gadler F, Kranig W, et al.; the TRIP-HF (Triple resynchronization in paced heart failure patients) study group. A randomized comparison of triple-site versus dual-site ventricular stimulation in patients with congestive heart failure. J Am Coll Cardiol 2008; 51: 1455-1462.

5. Lenarczyk R, Kowalski O, Sredniawa B, et al. Implantation feasibility, procedure-related adverse events and lead performance during 1-year follow-up in patients undergoing triple-site cardiac resynchronization therapy: a substudy of TRUST CRT randomized trial. J Cardiovasc Electrophysiol 2012; 23: 1228-1236.

6. Bordachar P, Alonso C, Anselme F, et al. Addition of a second LV pacing site in CRT nonresponders rationale and design of the multicenter randomized V(3) trial. J Card Fail 2010; 16: 709-713. 